\title{
Analytical Evaluation of Higher Order Sectorization, Frequency Reuse and User Classification Methods in OFDMA Networks
}

\author{
Jianhua He, Wenqing Cheng, Zuoyin Tang, David López-Pérez and Holger Claussen
}

\begin{abstract}
Higher order sectorization (HOS), which splits macrocells into a larger number of smaller sectors, are receiving significant interest as a cost-effective means of improving network capacity. Potentially, the capacity gain with HOS is proportionally linear to the number of sectors per cell due to spatial reuse, but factors such as non-ideal antenna radiation patterns together with inter-cell interference can significantly reduce this capacity gain. We develop a statistical model to theoretically characterize the performance of HOS deployments in wireless networks using orthogonal frequency division multiplex access (OFDMA). Moreover, a fractional frequency reuse scheme is considered, which aids to mitigate inter-cell interference. The model provides a fast and effective tool for studying network performance in terms of user signal quality, site throughput and outage probability, and it can be used to speed up network planning and optimization. In addition, we consider the impact of user classification methods in the analysis, and propose a new spectrum efficiency-based user classification method that improves resource utilization and allocation fairness. Performance results indicate that the proposed model is accurate, and shows a diminishing performance gain of HOS deployments with the number of antennas. The proposed user classification method improves network performances with respect to state of the art approaches.
\end{abstract}

\section{INTRODUCTION}

The increasing popularity of smartphones and video on demand is driving a strong growth in wireless network traffic. As a result, enhancing network capacity is a major concern of mobile operators. To do so, apart from well-known techniques such as small cell deployments, multi-antenna techniques, coordinated resource management and inter-cell interference mitigation, the usage of HOS is receiving significant interest in the cellular community [1]-[5]. With HOS deployments, macrocells are split into a larger number of smaller sectors by using of a large number of directional antennas per base station (BS). This facilitates spectrum reuse and increases the network capacity.

In the baseline configuration of cellular networks, macrocell BSs are usually equipped with 3 sectors. HOS with 6,12 or even more sectors per macrocell BS have the potential of enhancing network capacity by exploiting spatial reuse without

Jianhua He (email: j.he7@aston.ac.uk) and Zuoyin Tang (email: z.tangleaston.ac.uk) are with the School of Engineering and Applied Science, Aston University, UK. Wenqing Cheng (email: chengwqehust.edu.cn, Corresponding Author) is with the School of Electronic Information and Communications, Huazhong University of Science and Technology, China. David López-Pérez (email: david.lopez-perez@nokia.com) and Holger Claussen (email: holger.claussen@nokia.com) are with Bell Labs, Nokia, Ireland. incurring significant capital and operational expenditures compared to deploying new macrocell BSs or frequency carriers. However, HOS also come with their own challenges in terms of hardware development, inter-cell interference and mobility management, and there are a number of research questions that need to be addressed before HOS can be considered as a solid candidate technology for the next generation of cellular technologies.

The applicability of HOS to conventional macrocellular networks has been the subject of a large body of research in previous years, in both code division multiple access (CDMA) and OFDMA networks, e.g., [7]-[14].

In CDMA networks, the performance gain of HOS due to higher order diversity with fixed antennas beams was analysed in [7]. In [8], the performance of a real WCDMA network with 6-sector BSs and fixed antennas beams was evaluated under non-homogeneous network deployments. In [9], similar use cases as in [8] were investigated considering smart antennas.

In the last decade, OFDMA has been used in several cellular network and mobile broadband standards, including the downlink of the LTE and Mobile-WiMAX. Due to its different physical layer, the findings of [7]-[9] do not apply. In OFDMA networks, the performance gain of HOS with multiple antennas was evaluated in [10]. In [11], the HOS performance was compared with that of multi-user MIMO and network MIMO. In [12], this performance comparison was extended to include multi-user MIMO with 12 antennas per BS. Simulation results showed that HOS can achieve higher average user throughput than these MIMO techniques for some specific scenarios. Recently, in [13], the impact of antenna beamwidth on HOS performance was analysed. Moreover, in [14], a study of HOS performance in a LTE network using 3GPP compliant simulations was presented. In [15], a study of dynamic cell expansion with multiple sectors for energy efficiency improvement was presented, in which vertical sectorization was exploited.

The above research works indicate the potential of HOS, but only consider universal frequency reuse schemes and no intercell interference coordination techniques. As will be presented in Section V, HOS deployments with universal frequency reuse can lead to severe inter-cell interference and very high outage probability. In contrast, in OFDMA networks, fractional frequency reuse (FFR) schemes can be used to mitigate inter-cell interference, and have the potential to optimize the tradeoff between overall network and cell-edge performance [4], [19], [20]. The performance evaluation of HOS without interference 
mitigation techniques (such as frequency reuse schemes) may not provide proper recommendations for HOS deployments. Thus, we suggest that these two technologies should be developed and analysed in conjunction.

In addition to the mentioned issues, the above evaluations of HOS performance in OFDMA networks rely on traditional simulation based approaches that have been used in LTE networks [21]. In order to have an effective evaluation of HOS deployments, a fast and effective analytical tool for system level performance evaluation of HOS deployments with FFR schemes in OFDMA networks is highly demanded. Such analytical tool can also help achieving the research target of supporting the reproducability of performance evaluations conducted by different researchers [34].

In this paper, in view of the aforementioned research problems, we investigate HOS deployments with FFR schemes in OFDMA networks, and develop a novel statistical model to theoretically characterize their performance. The main contributions of this paper are summarized in the following:

- We have developed a unified and accurate statistical analytical model for the performance evaluation of HOS deployments with FFR schemes in OFDMA networks. In the proposed model, we concentrate on a strict FFR scheme [4], [19], [20], and apply the widely used FentonWilkinson method to approximate the sum of log-normal distributed interferers as log-normal distributed random variables (RVs) [24]. We also extend the analytical model to correlated interferers [25]. The proposed model provides a fast and effective tool for performance evaluation, and can be used to aid network planning and optimization procedures in HOS deployments. In the literature, many analytical models have been developed for mobile cellular networks including the increasingly popular stochastic geometry based models (e.g. [3], [5], [20], [22], [23], [30], [31]), but sector antenna radiation pattern and correlated shadowing have not been taken into account.

- We have investigated the impact of inter-cell interference mitigation in HOS deployments with FFR schemes in OFDMA networks in terms of overall network throughput and user outage probability. We propose a spectrum efficiency based user classification method for FFR schemes that improves resource utilization and allocation fairness in HOS deployments.

- With both analytical and simulation evaluation approaches, which provide cross verification and higher reliability, we have conducted a comprehensive performance evaluation of HOS deployments using an abstracted LTE link level model. The impacts of 3D antenna radiation pattern, FFR schemes and many other system parameters on the network performance (e.g. user signal quality, site throughput and outage probability) are considered. Different HOS, FFR and user classification combinations are compared. A diminishing performance gain with the number of antennas is observed. The numerical analysis of HOS deployments sheds some new light in this research area.

The rest of this paper is organized as follows. In Section II,

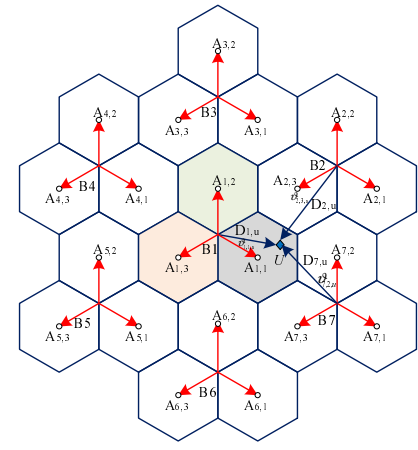

(a) 3-sectors

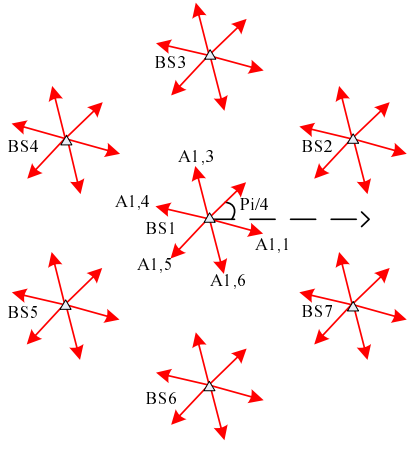

(b) 6-sectors.
Fig. 1. Examples of cellular network layouts.

the system model and assumptions are introduced. In Section III, various user classification methods for FFR schemes are presented. In Section IV, an analytical model is developed for HOS performance analysis with and without FFR schemes. In Section V, the numerical results and discussions are presented. In Section VI, the conclusions are drawn.

\section{SySTEM MODEL}

We consider the BS configurations with 3, 6 and 12 sectors. For the 3-sector BS configuration, a clover-leaf network layout is used ${ }^{1}$. It is important to note that the analytical model developed in this paper is general, and can also be used for higher order sectorization as well as other network deployment layouts.

Fig. 1(a) shows an example of the 3-sector BS hexagonal network layout with 1 tier of interfering BSs. For the 6sector and 12-sector BS configurations, the BSs are placed at identical locations, but the main directions of the sector antennas are offset with respect to the 3-sector layout to avoid neighbouring sectors pointing each other. Fig. 1(b) shows an example of 6-sector BS network layout. It is important to note that the analytical model developed in this paper is general, and can also be used for higher order sectorization as well as other network deployment layouts.

Using these network layouts, let us consider a cellular network with $N_{\text {sites }}$ sites equipped with one BS each, where the $i$ th BS $\left(i \in\left[1, N_{\text {sites }}\right]\right)$ is located at $B_{i}$. The inter-site distance is denoted by $R_{\mathrm{ISD}}$.

The number of sectors per BS is denoted by $N_{\mathrm{a}}$, and as indicated earlier, we concentrate our analyses on BS configurations with $N_{\mathrm{a}}=3, N_{\mathrm{a}}=6$ or $N_{\mathrm{a}}=12$. The $j$ th sector of the $i$ th site is denoted by $\mathcal{A}_{i, j}$, where $i \in\left[1, N_{\text {sites }}\right]$ and $j \in\left[1, N_{\mathrm{a}}\right]$. Let $\vartheta_{i, j}$ denote the horizontal angle of the main radiation direction of sector $\mathcal{A}_{i, j}$, which is set to $(j-1) * \pi / N_{\mathrm{a}}-\vartheta_{i, 1}$ for $\left(j \in\left[2, N_{\mathrm{a}}\right]\right)$, with $\vartheta_{i, 1}=-\pi / 6$ for the 3-sectors and $\vartheta_{i, 1}=-\pi / 12$ for the 6-sector and 12-sector BS configurations, respectively.

Users are assumed to be uniformly distributed within each service area. Without loss of generality, we focus our analysis on the performance of users associated with sector $\mathcal{A}_{1,1}$,

\footnotetext{
${ }^{1}$ For the 3-sector BS case, the clover-leaf network layout was found to have a better performance than the hexagonal network layout [16]
} 
and assume that BSs are equipped with a round-robin scheduler. We also consider a fully loaded network, in which the frequency resources allocated to each sector are fully used. Table 1 lists the main notations used in this paper.

\section{A. Channel model and antenna radiation pattern}

Since we consider a fully loaded network, we focus our studies on the transmission performance of one physical resource block (PRB), which represents the basic time-frequency resource unit available for data transmission in LTE networks.

Let us consider a general user $u$ located at position $L_{u}$ with coordinates $\left(x_{u}, y_{u}\right)$ inside sector $\mathcal{A}_{1,1}$. Let $D_{i, u}$ be the distance between BS $i$ and user $u$, and $\vartheta_{i, j, u}$ be the angle between the main radiation direction of sector $\mathcal{A}_{i, j}$ and user $u$, which can be calculated by basic geometric methods.

Let us now define the signal power $\mathcal{P}_{i, j, u}$ received by user $u$ from sector $\mathcal{A}_{i, j}$ as

$$
\mathcal{P}_{i, j, u}=P_{\mathrm{t}} G_{\mathrm{PL}}(i, u) \psi_{i, u} G_{\mathrm{A}}(i, j, u),
$$

where $P_{\mathrm{t}}$ is the BS transmit power over a PRB, $G_{\mathrm{PL}}(i, u)$ is the path gain (loss) between BS $i$ and user $u, \psi_{i, u}$ is the shadow fading between BS $i$ and user $u$, and $G_{\mathrm{A}}(i, j, u)$ is the antenna gain between sector $\mathcal{A}_{i, j}$ and user $u$, For ease of notation, we let $P_{\mathrm{r}, \mathrm{i}, \mathrm{j}, \mathrm{u}}=P_{\mathrm{t}} G_{\mathrm{PL}}(i, u) G_{\mathrm{A}}(i, j, u)$ denote the mean received power by user $u$ from sector $\mathcal{A}_{i, j}$.

The path gain (loss) $G_{\mathrm{PL}}(d)$ models the propagation loss between BS $i$ and user $u$. The model specified in [6] for outdoor line-of-sight communications is used,

$$
G_{\mathrm{PL}}(d)=-34.02-22 \log _{10}(\mathrm{~d})[\mathrm{dB}] .
$$

where $d$ is the distance between BS $i$ and user $u$.

The shadow fading $\psi_{i, u}$ models the variability of the path loss between BS $i$ and user $u$, which is assumed to follow a log-normal distribution with location value of 0 and scale value of $\sigma$ [6]. Moreover, the shadow fading within sectors of a site is assumed to be fully correlated, while the inter-site shadow fading correlation is denoted by $\rho$.

The antenna gain $G_{\mathrm{A}}(i, j, u)$ models the gain of the antenna in the direction between sector $\mathcal{A}_{i, j}$ and user $u$,

$$
G_{\mathrm{A}}(i, j, u)=G_{\mathrm{A}, \max } G_{\mathrm{A}, \mathrm{h}, \mathrm{v}}\left(\vartheta_{i, j, u}, \theta_{i, j, u}\right),
$$

where $G_{\mathrm{A}, \max }$ is the maximum antenna gain, and $G_{\mathrm{A}, \mathrm{h}, \mathrm{v}}(\vartheta, \theta)$ is the 3-dimensional antenna radiation pattern with horizontal angle $\vartheta$ and vertical angle $\theta$.

$G_{\mathrm{A}, \mathrm{h}, \mathrm{v}}(\vartheta, \theta)$ is calculated using the model in [6] as

$G_{\mathrm{A}, \mathrm{h}, \mathrm{v}}^{\mathrm{dB}}(\vartheta, \theta)=-\min \left(-1 *\left(G_{\mathrm{A}, \mathrm{h}}^{\mathrm{dB}}(\vartheta)+G_{\mathrm{A}, \mathrm{v}}^{\mathrm{dB}}(\theta)\right), G_{\mathrm{Front}}^{\mathrm{dB}}\right)$,

$G_{\mathrm{A}, \mathrm{h}, \mathrm{v}}(\vartheta, \theta)=10^{G_{\mathrm{A}, \mathrm{h}, \mathrm{v}}^{\mathrm{dB}}(\vartheta, \theta) / 10}$.

where $G_{\mathrm{A}, \mathrm{h}}\left(\vartheta_{i, j, u}\right)$ and $G_{\mathrm{A}, \mathrm{v}}\left(\theta_{i, j, u}\right)$ are the normalized horizontal and vertical radiation pattern offsets of the sector antenna, and $G_{\text {Front }}^{\mathrm{dB}}$ is the antenna front to back ratio.

Note that $G_{\mathrm{A}, \mathrm{h}}\left(\vartheta_{i, j, u}\right)$ and $G_{\mathrm{A}, \mathrm{v}}\left(\theta_{i, j, u}\right)$ are calculated as

$$
G_{\mathrm{A}, \mathrm{h}}^{\mathrm{dB}}(\vartheta)=-\min \left(12\left(\frac{\vartheta}{\vartheta_{3 \mathrm{~dB}}}\right)^{2}, 25\right),
$$

and

$$
G_{\mathrm{A}, \mathrm{v}}^{\mathrm{dB}}(\theta)=-\min \left(12\left(\frac{\theta-\theta_{\mathrm{down}}}{\theta_{3 \mathrm{~dB}}}\right)^{2}, 20\right),
$$

where $\vartheta_{3 \mathrm{~dB}}$ and $\theta_{3 \mathrm{~dB}}$ are the horizontal and vertical halfpower beamwidth (HPBW), and $\theta_{\text {down }}$ is the down-tilt angle.

Taking into account the previous definitions, the signal to interference plus noise ratio (SINR) $\gamma_{u}$ of user $u$ located at $\left(x_{u}, y_{u}\right)$ within sector $\mathcal{A}_{1,1}$ can be calculated as

$$
\gamma_{u}=\frac{\mathcal{S}_{u}}{\sum_{j \in \Omega_{\text {Intra }, u}} \mathcal{P}_{1, j, u}+\sum_{i=2}^{N_{\text {sites }}} \sum_{j \in \Omega_{\text {Inter }, u}} \mathcal{P}_{i, j, u}+\delta^{2}}, \text { (7) }
$$

where $\mathcal{S}_{u} \equiv \mathcal{P}_{i, j, u}$ is the designated signal for user $u, \Omega_{\text {Intra }, u}$ and $\Omega_{\text {Inter, } u}$ are the set of IDs of intra-site and inter-site interfering sectors with regard to user $u$, respectively, which are determined by the used frequency reuse scheme, and $\delta^{2}$ is the noise power, which can be calculated as the product of the noise figure and the signal bandwidth. With the assumption of a fully loaded network, the downlink communication is generally interference limited, therefore the impact of noise is negligible and is not considered in this paper.

It is important to note that $\mathcal{P}_{i, j, u}$ is a log-normal variable, and can be uniquely characterized by its mean and variance, which are denoted by $\overline{\mathcal{P}_{i, j, u}}$ and $\widehat{\mathcal{P}_{i, j, u}}$, respectively.

\section{USER ClassificATION FOR Fractional FREQUENCY REUSE SCHEMES}

In this paper, we considered a strict FFR in this paper. However, it is noted that the proposed user classification method and analytical model are not limited to the strict FFR scheme, but can work with other variants of FFR schemes, such as soft FFR schemes [4]. In the strict FFR, the available bandwidth (denoted as $W$ ) is divided into two sub-bands, cell-centre and cell-edge sub-bands, (the bandwidth of which are denoted as $W_{\mathrm{c}}$ and $W_{\mathrm{e}}$, respectively), where $\beta$ is the bandwidth partition coefficient, i.e., $W_{\mathrm{c}}=\beta W$ and $W_{\mathrm{e}}=(1-\beta) W$. Moreover, cell-centre sub-bands use a frequency reuse factor of 1 (IFR1), while cell-edge sub-bands use a frequency reuse of factor 3 (IFR-3). An identical downlink transmit power is applied to both sub-bands.

Fig. 2 shows an example of FFR bandwidth reuse for cellcentre and cell-edge in 3-sectors and 6-sectors configurations.

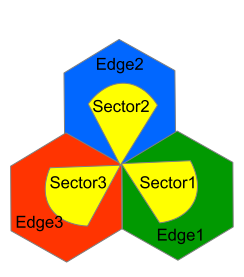

(a) 3-sectors

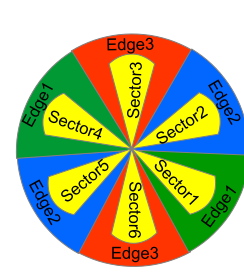

(b) 6-sectors

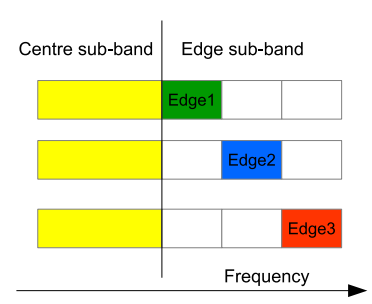

(c) bandwidth partitioning
Fig. 2. Illustration of FFR bandwidth reuse with 3-sectors and 6-sectors configurations.

It is noted that the shapes of sector coverage shown in Fig. 2(a) and Fig. 2(b) are given for illustration only. The actual shapes are determined by many factors such as antenna directions, antenna patterns and shadowing. With this bandwidth partitioning, cell-centre users can only access the 
TABLE I

NOTATIONS

\begin{tabular}{|c|c|c|c|}
\hline Notation & Meaning & Notation & Meaning \\
\hline $\begin{array}{l}N_{\text {sites }} \\
\mathcal{A}_{i, j} \\
A_{i, j} \\
D_{i, u} \\
\mathcal{P}_{i, j, u} \\
G_{\mathrm{PL}}(i, u) \\
\psi_{i, u} \\
G_{\mathrm{A}, \max } \\
\vartheta_{3 \mathrm{~dB}}, \theta_{3 \mathrm{~dB}} \\
\sigma_{\mathrm{w}} \\
\gamma_{u}, \mathcal{S}_{u} \\
W, W_{\mathrm{c}}, W_{\mathrm{e}} \\
\gamma_{\mathrm{c}, u}, \gamma_{\mathrm{e}, u} \\
I_{\mathrm{c}, i, u} \\
I_{\mathrm{sum}, \mathrm{c}, u}\end{array}$ & $\begin{array}{l}\text { number of sites } \\
j \text { th sector of } i \text { th site } \\
\text { centre location of sector } \mathcal{A}_{i, j} \\
\text { distance between BS } i \text { and user } u \\
\text { power received by user } u \text { from } \mathcal{A}_{i, j} \\
\text { path loss between BS } i \text { and user } u \\
\text { shadow fading between BS } i \text { and } u \\
\text { maximum antenna gain } \\
\text { horizontal and vertical HPBW } \\
\text { log-normal shadowing standard deviation } \\
\text { user SIR, designated user signal } \\
\text { available, cell-centre and cell-edge BW } \\
\text { SIRs of cell-centre, cell-edge user } u \\
\text { sum of interference from BS } i \text { to } u \\
\text { aggregate interference from cells to } u\end{array}$ & $\begin{array}{l}R_{\mathrm{ISD}} \\
N_{\mathrm{a}} \\
L_{u} \\
\vartheta_{i, j, u} \\
P_{\mathrm{t}} \\
G_{\mathrm{A}}(i, j, u) \\
P_{\mathrm{r}, \mathrm{i}, \mathrm{j}, \mathrm{u}} \\
G_{\mathrm{A}, \mathrm{h}, \mathrm{v}}(\vartheta, \theta) \\
\psi_{i, u} \\
\rho \\
\gamma_{\mathrm{net}}, \mathcal{X}_{\mathrm{site}}, \mathcal{O}_{\mathrm{net}} \\
\beta \\
\mathcal{X}_{\mathrm{c}, u}, \mathcal{X}_{\mathrm{e}, u} \\
\mu_{I_{\mathrm{c}, i, u}}, \sigma_{I_{\mathrm{c}, i, u}} \\
\mu_{I_{\mathrm{I}, u m}}, \sigma_{I_{\mathrm{s} u m}}\end{array}$ & $\begin{array}{l}\text { inter-site distance } \\
\text { number of sectors per BS } \\
\text { location of user } u \\
\text { angle between sector } \mathcal{A}_{i, j} \text { and user } u \\
\text { BS transmit power over one PRB } \\
\text { antenna gain between } \mathcal{A}_{i, j} \text { and user } u \\
\text { mean received power by } u \text { from } \mathcal{A}_{i, j} \\
\text { antenna radiation pattern with } \vartheta, \theta \\
\text { shadowing between BS } i \text { and user } u \\
\text { inter-site shadow fading correlation } \\
\text { network SIR, throughput, outage prob. } \\
\text { bandwidth partition coefficient } \\
\text { cell-centre, cell-edge user throughput } \\
\text { mean and std of } I_{\mathrm{c}, i, u} \\
\text { mean and std of } I_{\mathrm{sum}, \mathrm{c}, u}\end{array}$ \\
\hline
\end{tabular}

cell-centre sub-bands and cell-edge users can only access the cell-edge sub-bands.

\section{A. Distance-based user classification (UC-Dist) method}

With the distance-based classification method, a user is classified as cell-centre user if its distance to the serving BS is less than a given distance threshold, i.e, $\alpha R_{\mathrm{ISD}} / 2$, where $\alpha$ is the so-called distance coefficient, $0 \leq \alpha \leq 1$; otherwise it is classified as cell-edge user. In the following, this user classification method is abbreviated as UC-Dist $\alpha$ method. It is noted that IFR-1 and IFR-3 schemes can be regarded as two specific cases of the $U C$-Dist $\alpha$ method, where $\alpha$ is set to 1 and 0 , respectively.

This method however has some drawbacks.

- As user position information is required, this method may have limited application in real cellular networks.

- For BSs with omni-directional antennas, a smaller distance between the serving BS and the user usually means better SIR and therefore improved spectrum efficiency. However, this is not always the case for BSs with sector antennas. The $U C$-Dist $\alpha$ method may thus result in a low spectrum efficiency when considering sector antennas.

- Another potential issue is that the proportion of traffic generated by the users in the centre and edge zones may quickly change in the time domain due to user mobility, traffic patterns, etc. This may lead to an unfair frequency resource sharing between cell-centre and cell-edge users.

\section{B. RSS based user classification (UC-RSS) method}

In [18], an alternative user classification method based on user relative RSS was proposed. With the RSS-based classification method, a user is classified as cell-centre user, if the received signal strength from the best serving cell is $3 \mathrm{~dB}$ higher than that received signal strength from the strongest neighbouring cell; otherwise it is classified as cell-edge user. In the following, this user classification method is abbreviated as $U C$-RSS $\alpha$ method.

The UC-RSS method solves the problems faced by the $U C$-Dist $\alpha$ method when using sector antennas, but suffers from the same unfair frequency resource sharing and mobility issues.

\section{Spectrum efficiency-based user classification (UC-Spe) methods}

As both distance- and RSS-based methods may not be responsive enough to cope with traffic fluctuations when having a fixed bandwidth partition for cell-centre and cell-edge users, we propose a spectrum efficiency-based user classification method for FFR schemes, with the following objectives:

1) To maximize spectrum efficiency of the entire network.

2) To maximize the resource allocation fairness between cell-centre and cell-edge users.

3) To minimize the overhead, and enhance the flexibility. Within the cell-centre and cell-edge zones, various resource partition and scheduling algorithms may be used.

In the proposed user classification method, we first estimate the SIR and the throughput of users as both cell-centre and cell-edge users, and then decide which users should be classified as cell-centre and cell-edge users, by the following procedure.

Suppose there are $N_{\text {user }}$ active users in sector $\mathcal{A}_{1,1}$, and let $\Omega_{\mathrm{c}}$ and $\Omega_{\mathrm{e}}$ respectively denote the sets of cell-centre and celledge users after the prior user classification procedure completes, and $N_{\mathrm{c}}$ and $N_{\mathrm{e}}$ respectively denote their cardinalities, where $N_{\text {user }}=N_{\mathrm{c}}+N_{\mathrm{e}}$. In order to achieve fairness, ideally the number of users in each set should be proportional to the bandwidth of each sub-band, i.e.,

$$
\frac{W_{\mathrm{c}}}{N_{\mathrm{c}}}=\frac{W_{\mathrm{e}}}{N_{\mathrm{e}}}
$$

where $N_{\mathrm{c}}=\left\lceil\beta N_{\text {user }}\right\rceil$, as the number of users needs to be an integer. It is noted that the users within a given group are assumed to have similar bandwidth requirements.

The proposed resource sharing criterion in (8) can be changed to achieve a given tradeoff between fair spectrum allocation and throughput with the introduction of a resource sharing control variable $w_{\mathrm{c}, \mathrm{e}}$, which can be defined as

$$
\frac{W_{\mathrm{c}}}{N_{\mathrm{c}}}=\frac{w_{\mathrm{c}, \mathrm{e}} W_{\mathrm{e}}}{N_{\mathrm{e}}}
$$

where $w_{\mathrm{c}, \mathrm{e}}=1$ in this paper for the sake of simplicity.

Let $\gamma_{\mathrm{c}, \mathrm{u}}$ and $\gamma_{\mathrm{e}, \mathrm{u}}$ denote the SIRs of user $u$ as cell-centre and cell-edge user, respectively, and let $\mathcal{X}_{\mathrm{c}, u}$ and $\mathcal{X}_{\mathrm{e}, u}$ denote 
the throughput that can be achieved by user $u$ as cell-centre and cell-edge user, respectively. Moreover, let $\mathcal{F}(x)$ define a function to calculate the user capacity per unit of bandwidth based on the user SIR $x$ (The proposed user classification method can work with different user capacity functions).

According to previous definitions and assuming a round robin scheduler, the user throughput as cell-centre and celledge user can be calculated as

$$
\begin{aligned}
& \mathcal{X}_{\mathrm{c}, \mathrm{u}}=\frac{\mathcal{F}\left(\overline{\gamma_{\mathrm{c}, \mathrm{u}}}\right) W_{\mathrm{c}}}{N_{\mathrm{c}}}, \\
& \mathcal{X}_{\mathrm{e}, \mathrm{u}}=\frac{\mathcal{F}\left(\overline{\gamma_{\mathrm{e}, \mathrm{u}}}\right) W_{\mathrm{e}}}{3 N_{\mathrm{e}}} .
\end{aligned}
$$

Then, the site throughput can be calculated as the multiplication of the number of sectors $\left(N_{\mathrm{a}}\right)$ and the sector throughput from (13)

$$
\begin{aligned}
\mathcal{X}_{\text {site }} & =N_{\mathrm{a}}\left(\sum_{u \in \Omega_{\mathrm{c}}} \mathcal{X}_{\mathrm{c}, u}+\sum_{u \in \Omega_{\mathrm{e}}} \mathcal{X}_{\mathrm{e}, u}\right) \\
& =N_{\mathrm{a}}\left[\sum_{u \in \Omega_{\mathrm{c}}} \frac{\mathcal{F}\left(\overline{\gamma_{\mathrm{c}, \mathrm{u}}}\right) W_{\mathrm{c}}}{N_{\mathrm{c}}}+\sum_{u \in \Omega_{\mathrm{e}}} \frac{\mathcal{F}\left(\overline{\gamma_{\mathrm{e}, \mathrm{u}}}\right) W_{\mathrm{e}}}{3 N_{\mathrm{e}}}\right] \\
& \approx N_{\mathrm{a}}\left[\frac{W}{N_{\mathrm{user}}} \sum_{u \in \Omega_{\mathrm{c}}} \mathcal{F}\left(\overline{\gamma_{\mathrm{c}, \mathrm{u}}}\right)+\frac{W}{3 N_{\mathrm{user}}} \sum_{u \in \Omega_{\mathrm{e}}} \mathcal{F}\left(\overline{\left.\gamma_{\mathrm{e}}, \mathrm{b} \mathrm{l}\right)}\right)\right] \\
& =\frac{N_{\mathrm{a}} W}{3 N_{\mathrm{user}}}\left[\sum_{u \in \Omega_{\mathrm{c}}} 3 \mathcal{F}\left(\overline{\gamma_{\mathrm{c}, \mathrm{u}}}\right)+\sum_{u \in \Omega_{\mathrm{e}}} \mathcal{F}\left(\overline{\gamma_{\mathrm{e}, \mathrm{u}}}\right)\right] .
\end{aligned}
$$

Let $\mathcal{X}_{\mathrm{c}-\mathrm{e}, u}$ also define a new variable referred to as throughput difference that represents the difference between $\mathcal{X}_{\mathrm{c}, u}$ and $\mathcal{X}_{\mathrm{e}, u}$ for user $u$, which is calculated as

$$
\mathcal{X}_{\mathrm{c}-\mathrm{e}, u}=\mathcal{X}_{\mathrm{c}, u}-\mathcal{X}_{\mathrm{e}, u}
$$

According to (13), in order to maximize $\mathcal{X}_{\text {site }}$, the users with larger throughput difference should be chosen as cellcentre users with higher priority. We thus sort the set of users according to $\mathcal{X}_{\mathrm{c}-\mathrm{e}, \mathrm{u}}$ for $u=1, \ldots, N_{\mathrm{user}}$ in decreasing order, and then choose the first $N_{\mathrm{c}}$ users as cell centre-users and the remaining $N_{\text {user }}-N_{\text {c }}$ users as cell-edge users. As a result, we avoid a fixed user to bandwidth mapping as in the $U C$-Dist and $U C$-RSS methods.

It is noted that in the above method, the actual mean SIR is used in the user throughput calculation. This method with accurate SIR is abbreviated as UC-Spe-Acc method. Algorithm 1 presents the pseudocode of the proposed $U C$ Spe-Acc method.

\section{Algorithm 1 User classification method UC-Spe-Acc}

Input: $A$ set $\Omega_{\mathrm{U}}$ of users to be classified; Bandwidth partition coefficient $\beta$;

Output: A set $\Omega_{\mathrm{c}}$ of center users and a set $\Omega_{\mathrm{e}}$ of edge user;

$1: \Omega_{\mathrm{c}}=\phi, \Omega_{\mathrm{e}}=\phi$;

2: Calculate the expected number of cell-centre users $N_{\mathrm{c}}$ by (8).

3: Each user $u$ estimates $\overline{\gamma_{\mathrm{c}, u}}$ and $\overline{\gamma_{\mathrm{e}, u}}$ by measurement;

4: Calculate $\mathcal{X}_{\mathrm{c}, u}, \mathcal{X}_{\mathrm{e}, u}$ and $\mathcal{X}_{\mathrm{c}-\mathrm{e}, u}$ for $u \in \Omega_{\mathrm{U}}$;

5: Each user $u$ reports $\mathcal{X}_{\mathrm{c}-\mathrm{e}, u}$ to the serving BS.

6: The serving BS sorts $\mathcal{X}_{\mathrm{c}-\mathrm{e}, u}\left(u \in \Omega_{\mathrm{U}}\right)$ in decreasing order;
7: Add the first $N_{\mathrm{c}}$ users in the sorted set to the set of cell-centre users $\Omega_{\mathrm{c}}$; add the remaining users to the set of cell-edge users $\Omega_{\mathrm{e}}$.

8: Perform scheduling.

9: Return.

In order to simplify the SINR classification process, we propose an alternative user classification method in which an approximated SIR instead of an actual SIR is used. In this approximation, the RSS from the cell that generates the maximum interference to the user is used to calculate the approximated interference and SIR $\widetilde{\gamma}_{\mathrm{c}, u}$ and $\widetilde{\gamma}_{\mathrm{e}, u}$ of user $u$ as a cell-centre and cell-edge user:

$$
\begin{aligned}
& \widetilde{\gamma}_{\mathrm{c}, u}=\frac{P_{\mathrm{r}, 1,1, u}}{C_{\mathrm{app}} \times \max \left\{\sum_{j=1}^{N_{\mathrm{a}}} P_{\mathrm{r}, i, j, u} \mid i=2, \ldots, N_{\text {sites }}\right\}^{(15)}} \\
& \widetilde{\gamma}_{\mathrm{e}, u}=\frac{P_{\mathrm{r}, 1,1, u}}{C_{\mathrm{app}} \times \max \left\{P_{\mathrm{r}, i, 1, u} \mid i=1, \ldots, N_{\text {sites }}\right\}} .
\end{aligned}
$$

In the above equations, the maximum single cell interference to user $u$ is multiplied by a coefficient $C_{\text {app }}$ to approximate the overall network interference. After sampling the values in the range $[1,2.5]$ for coefficient $C_{\text {app }}$ in the studied scenarios, we set $C_{\text {app }}$ to 1.5 , and implement an alternative user classification method following the procedure shown in Algorithm 1. This method with approximated SIR is abbreviated as UC-SpeApp method. Note that the SIR approximation is used only in the user classification process. The actual SIR is used in the network performance evaluation by simulation and analytical approaches.

The spectrum efficiency user classification methods are viable. The core of the spectrum efficiency based user classification method is on the measurement of SIR. Usually the SIR and throughput are dominated by a few close interfering sectors/BSs. As specified by 3GPP UEs are required to periodically measure and report the reference signal received power from the neighbour cells for cell search and hand over purposes [1], they are readily available for the user classification process. The time resolution of the proposed classification method can be set as that used for user handover.

\section{Analytical Performance Model for FRACTIONAL FREQUENCY REUSE SCHEMES}

As indicated in Section II, we focus our analysis on the performance of users associated with sector $\mathcal{A}_{1,1}$, based on which the overall network performance can be calculated. With the above user classification strategies, a user located anywhere within the service area of sector $\mathcal{A}_{1,1}$ can be classified as cellcentre or cell-edge user.

In this section, we first derive a statistical model for deriving the SIR distribution of an individual user $u$ located at $\left(x_{u}, y_{u}\right)$ as a cell-centre user as well as a cell-edge user. With this model for individual users, we then calculate network performance in terms of network throughput and outage probabilities for different FFR schemes.

\section{A. SIR distribution for a cell-centre user}

As an IFR-1 scheme is applied in the cell-centre sub-band, a cell-centre user within sector $\mathcal{A}_{1,1}$ is subject to interference 
from the sectors of BS 1 other than sector 1 as well as all sectors of all neighbouring cells. Therefore, for a cell-centre user $u$ of sector $\mathcal{A}_{1,1}$, we have the following expressions for the sets of IDs of intra-site and inter-site interfering sectors

$$
\begin{aligned}
\Omega_{\text {Intra }, u} & =\left\{j \mid 2 \leq j \leq N_{\mathrm{a}}\right\} \\
\Omega_{\text {Inter }, u} & =\left\{j \mid 1 \leq j \leq N_{\mathrm{a}}\right\} .
\end{aligned}
$$

According to (7), the SIR $\gamma_{\mathrm{c}, u}$ of user $u$ within sector $\mathcal{A}_{1,1}$ as a cell-centre user can be calculated as

$$
\begin{aligned}
\gamma_{\mathrm{c}, u} & =\frac{P_{\mathrm{r}, 1,1, u} \psi_{1, u}}{\sum_{j \in \Omega_{\mathrm{Intra}, u}} P_{\mathrm{r}, 1, j, u} \psi_{1, u}+\sum_{i=2}^{N_{\mathrm{sites}}} \sum_{j \in \Omega_{\mathrm{Inter}, u}} P_{\mathrm{r}, i, j, u} \psi_{i, u}} \\
& =\frac{P_{\mathrm{r}, 1,1, u} \psi_{1, u}}{\sum_{i=1}^{N_{\text {sites }}} I_{\mathrm{c}, i, u}}
\end{aligned}
$$

where $I_{\mathrm{c}, i, u}$ is the sum of the interference generated from the different sectors of the $i$ th BS to user $u$, i.e.,

$$
I_{\mathrm{c}, i, u}= \begin{cases}\sum_{j \in \Omega_{\text {Intra }, u}} P_{\mathrm{r}, 1, j, u} \psi_{1, u}, & i=1, \\ \sum_{j \in \Omega_{\text {Inter }, u}} P_{\mathrm{r}, i, j, u} \psi_{i, u}, & i=2, \ldots, N_{\text {sites }} .\end{cases}
$$

Note that $I_{\mathrm{c}, i, u}$ is a $\log$-normal RV as it has been assumed that the shadow fading within sectors of a site is fully correlated in Section II.

Let $\mu_{I_{\mathrm{c}, i, u}}$ and $\sigma_{I_{\mathrm{c}, i, u}}$ denote the mean and the standard deviation of the normal distribution associated with $I_{\mathrm{c}, i, u}$, respectively, which can be calculated by

$$
\mu_{I_{\mathrm{c}, i, u}}=\left\{\begin{array}{l}
\ln \left(\sum_{j=2}^{N_{\mathrm{a}}} P_{\mathrm{r}, i, j, u}\right) \\
\ln \left(\sum_{j=1}^{N_{\mathrm{a}}} P_{\mathrm{r}, i, j, u}\right) \\
i=2, \ldots, N_{\mathrm{sites}},
\end{array}\right.
$$

and

$$
\sigma_{I_{\mathrm{c}, i, u}}=\sigma_{\mathrm{w}} .
$$

Let us also define two new variables. $Y_{1, \mathrm{c}, u}=\frac{\sum_{j=2}^{N_{\mathrm{a}}} P_{\mathrm{r}, 1, j, u}}{P_{\mathrm{r}, 1,1, u}}$, and $Y_{\text {sum }, \mathrm{c}, u}$, which is the interference from neighbouring cells to signal ratio for cell-centre user $u$, i.e.,

$$
Y_{\text {sum }, \mathrm{c}, u}=\frac{I_{\text {sum }, \mathrm{c}, u}}{\mathcal{S}_{u}},
$$

where $I_{\mathrm{sum}, \mathrm{c}, u}$ is the sum of the interference from the neighbouring cells to cell-centre user $u$, and can be calculated as $I_{\text {sum }, \mathrm{c}, u}=\sum_{i=2}^{N_{\text {sites }}} I_{\mathrm{c}, i, u}$. It is important to note that $Y_{1, \mathrm{c}, u}$ is a deterministic variable, while $Y_{\mathrm{sum}, \mathrm{c}, u}$ is a RV.

Based on these two variables, the SIR $\gamma_{c, u}$ for cell-centre user $u$ within sector $\mathcal{A}_{1,1}$ can be expressed as

$$
\gamma_{\mathrm{c}, u}=\frac{1}{Y_{1, \mathrm{c}, u}+Y_{\mathrm{sum}, \mathrm{c}, u}} .
$$

In order to calculate the mean of $\gamma_{c, u}$, the sum of the interference $I_{\text {sum,c, } u}$ of the $N_{\text {sites }}-1$ correlated neighbouring cells to user $u$ is approximated by another log-normal variable, where $\mu_{I_{\text {sum }, \text {, }, u}}$ and $\sigma_{I_{\text {sum }, \text {, }, u}}$ denote the mean and standard deviation of the normal distribution associated with the lognormal approximation $I_{\mathrm{sum}, \mathrm{c}, u}$, respectively. Then, we can apply the method used in [26] to calculate the sum power of correlated log-normal RVs. The method has a lower complexity and still an acceptable accuracy compared to several other approximation methods [27]-[29]. According to such method, $\mu_{I_{\mathrm{sum}, \mathrm{c}, u}}$ can be calculated as

$$
\mu_{I_{\mathrm{sum}, \mathrm{c}, u}}=\log \left(\sum_{i=1}^{N_{\mathrm{sites}}} \mathrm{e}^{\mu_{I_{\mathrm{c}, i, u}}+0.5 \sigma_{I_{\mathrm{c}, i, u}}^{2}}\right)-\frac{\sigma_{I_{\mathrm{c}, i, u}}^{2}}{2}
$$

and $\sigma_{I_{\mathrm{sum}, \mathrm{c}, u}}$ can be calculated using (26).

$$
\begin{aligned}
\sigma_{I_{\mathrm{sum}, \mathrm{c}, u}}^{2} & =\log \left[1+\frac{\text { PARTA }+ \text { PARTB }}{\left(\sum_{i=2}^{N_{\mathrm{sites}}} \mathrm{e}^{\mu_{I_{\mathrm{c}, i, u}}^{2}+0.5 \sigma_{I_{\mathrm{c}, i, u}}^{2}}\right)^{2}}\right] \\
\text { PARTA } & \equiv \sum_{i=2}^{N_{\text {sites }}}\left(\mathrm{e}^{\left.\sigma_{I_{\mathrm{c}, i, u}}^{2}-1\right) \mathrm{e}^{2 \mu_{I_{\mathrm{c}, i, u}}+\sigma_{I_{\mathrm{c}, i, u}}^{2}}}\right. \\
\text { PARTB } & \equiv 2 \sum_{i=2}^{N_{\mathrm{sites}}-1} \sum_{j=i+1}^{N_{\mathrm{sites}}} \mathrm{e}^{\mu_{I_{\mathrm{c}, i, u}}+0.5 \sigma_{I_{\mathrm{c}, i, u}}^{2}} \mathrm{e}^{\mu_{I_{\mathrm{c}, j, u}}+0.5 \sigma_{I_{\mathrm{c}, j, u}}^{2}}\left(\mathrm{e}^{\rho \sigma_{I_{\mathrm{c}, i, u}}}\right.
\end{aligned}
$$

With the approximation of $I_{\text {sum,c, } u}$ as a log-normal variable and because $\mathcal{S}_{u}$ is also a log-normal variable, $Y_{\text {sum,c, } u}$ in the form of (23) is also known to be a log-normal variable, where $\mu_{Y_{\text {sum }, \mathrm{c}, u}}$ and $\sigma_{Y_{\text {sum }, \mathrm{c}, u}}$ denote the mean and standard deviation of the normal distribution associated with the lognormal variable $Y_{\text {sum }, \mathrm{c}, u}$, respectively.

As $I_{\text {sum }, \mathrm{c}, u}$ and $\mathcal{S}_{u}$ are correlated, let $\rho_{I_{\mathrm{sum}}, \mathcal{S}, \mathrm{c}, u}$ denote the correlation coefficient between $I_{\mathrm{sum}, \mathrm{c}, u}$ and $\mathcal{S}_{u}$. Then, we can write that

$$
\begin{aligned}
\mu_{Y_{\mathrm{sum}, \mathrm{c}, u}} & =\mu_{I_{\mathrm{sum}, \mathrm{c}, u}}-\ln \left(P_{\mathrm{r}, 1,1, \mathrm{u}}\right) \\
\sigma_{Y_{\mathrm{sum}, \mathrm{c}, u}}^{2} & =\sigma_{I_{\mathrm{sum}, \mathrm{c}, u}}^{2}+\sigma_{\mathrm{w}}^{2}-2 \rho_{\mathrm{sum}, 1, \mathrm{c}, u} \sigma_{I_{\mathrm{sum}, \mathrm{c}, u}} \sigma_{\mathrm{w}} .
\end{aligned}
$$

In this case, the only unknown variable to calculate $\mu_{Y_{\mathrm{sum}, \mathrm{c}, u}}$ and $\sigma_{Y_{\text {sum }, \mathrm{c}, u}}$ is $\rho_{I_{\mathrm{sum}}, \mathcal{S}, \mathrm{c}, u}$. We can use the method proposed in [33] to calculate $\rho_{\mathrm{sum}, \mathcal{S}, \mathrm{c}, u}$

$$
\begin{aligned}
\rho_{I_{\mathrm{sum}, \mathcal{S}, \mathrm{c}, u}}= & \frac{2\left[\ln (\nu)-\left(\mu_{I_{\mathrm{sum}, \mathrm{c}, u}}-\mu_{S_{u}}\right)\right]-\left(\sigma_{I_{\mathrm{sum}, \mathrm{c}, u}}^{2}+\sigma_{\mathrm{w}}^{2}\right)}{2 \sigma_{I_{\mathrm{sum}, \mathrm{c}, u}} \sigma_{\mathrm{w}}}, \\
\nu & =\sum_{i=2}^{N_{\mathrm{sites}}} \mathrm{e}^{\mu_{S_{u}}+\mu_{I_{\mathrm{c}, i, u}}+0.5\left(\sigma_{\mathrm{w}}^{2}+\sigma_{I_{\mathrm{sum}, \mathrm{c}, u}}^{2}+2 \rho \sigma_{\mathrm{w}} \sigma_{I_{\mathrm{sum}, \mathrm{c}, u}}\right)} .
\end{aligned}
$$

After we have calculated the mean $\mu_{Y_{\mathrm{sum}, \mathrm{c}, u}}$ and standard deviation $\sigma_{Y_{\text {sum }, \mathrm{c}, u}}$ of the normal distribution associated with log-normal variable $Y_{\text {sum }, c, u}$, the probability density function of $\gamma_{c, u}$ given by (24) can be determined accordingly. It is noted that for a general log-normally distributed RV $X$, with parameters $\mu_{X}$ and $\sigma_{X}$ being the mean and standard deviation of $X$ 's natural logarithm, the probability density function (denoted by $f_{X}\left(x ; \mu_{X}, \sigma_{X}\right)$ ) of $X$ can be expressed as

$$
f_{X}\left(x ; \mu_{X}, \sigma_{X}\right)=\frac{1}{x \sigma_{X} \sqrt{2 \pi}} \mathrm{e}^{-\frac{\left(\ln x-\mu_{X}\right)^{2}}{2 \sigma^{2}}} .
$$


Then, the mean of $\gamma_{\mathrm{c}, u}$ denoted by $\overline{\gamma_{\mathrm{c}, u}}$ for cell-centre user $u$ can be calculated as

$\overline{\gamma_{c, u}}=\int_{0}^{\infty} \frac{1}{\left(Y_{1, \mathrm{c}, u}+y\right)\left(y \sigma_{Y_{\mathrm{sum}, c, u}} \sqrt{2 \pi}\right)} \mathrm{e}^{-\frac{\left[\ln (y)-\mu_{Y_{\mathrm{sum}, c, u}}\right]^{2}}{2 \sigma_{Y_{\mathrm{sum}, c}, u}}}$

where $\overline{\gamma_{c, u}}$ can be obtained by simple numerical integration tools according to (32).

Based on the previous derivations, the throughput $\mathcal{X}_{c, u}$ that can be achieved by user $u$ as a cell-centre user can be calculated by (10).

a) Outage probability: Let us now define outage probability as the probability that the instantaneous SIR of a user is less than a given threshold. Then, the outage probability $\mathcal{O}_{c, u, \gamma_{\text {thr }}}$ for a cell-centre user $u$ with a given SIR threshold $\gamma_{\text {thr }}$ can be calculated as

$$
\begin{aligned}
\mathcal{O}_{\mathrm{c}, u, \gamma_{\mathrm{thr}}} & =\operatorname{Prob}\left(\gamma_{\mathrm{c}, \mathrm{u}}<\gamma_{\mathrm{thr}}\right) \\
& =\operatorname{Prob}\left(\frac{1}{\mathrm{Y}_{1, \mathrm{c}, \mathrm{u}}+\mathrm{Y}_{\mathrm{sum}, \mathrm{c}, \mathrm{u}}}<\gamma_{\mathrm{thr}}\right) \\
& =\int_{Y_{\mathrm{thr}, \mathrm{c}, u}}^{\infty} \frac{1}{y \sigma_{Y_{\mathrm{sum}, c, u}} \sqrt{2 \pi}} \mathrm{e}^{-\frac{\left[\ln (y)-\mu_{\left.Y_{\mathrm{sum}, c, u}\right]^{2}}\right.}{2 \sigma_{Y_{\mathrm{sum}, \mathrm{c}, u}}}} d y \\
& =1-\Phi\left(\frac{\ln \left(Y_{\mathrm{thr}, \mathrm{c}, u}\right)-\mu_{Y_{\mathrm{sum}, c, u}}}{\sigma_{Y_{\mathrm{sum}, c, u}}}\right)
\end{aligned}
$$

where $Y_{\mathrm{thr}, \mathrm{c}, u}=\frac{1}{\gamma_{\mathrm{thr}}}-Y_{1, \mathrm{c}, u}$ and $\Phi(x)$ is the cumulative distribution function of the standard normal distribution.

\section{B. SIR distribution for a cell-edge user}

As an IFR-3 scheme is applied in the cell-edge sub-band, a cell-edge user within sector $\mathcal{A}_{1,1}$ is subject to interference from a subset of sectors. Therefore, for a cell-edge user $u$ of sector $\mathcal{A}_{1,1}$, we have the following expressions for the sets of IDs of intra-site and inter-site interfering sectors

$$
\begin{aligned}
& \Omega_{\text {Intra }, u}=\left\{j \mid j=3 k+1,1 \leq k \leq\left\lfloor\frac{N_{\mathrm{a}}}{3}\right\rfloor\right\}, \\
& \Omega_{\text {Inter }, u}=\left\{j \mid j=3 k+1,0 \leq k \leq\left\lfloor\frac{N_{\mathrm{a}}}{3}\right\rfloor\right\} .
\end{aligned}
$$

Let $\gamma_{\mathrm{e}, u}$ denote the SIR of user $u$ as a cell-edge user, and $I_{\mathrm{e}, i, u}$ denote the sum of the interference generated from the different sectors of the $i$ th BS to user $u$. It is important to note that apart from the expressions of the sets of IDs of the intra-site and inter-site interfering sectors for cell-centre and cell-edge users, the expressions of SIR $\gamma_{\mathrm{e}, u}$ and $I_{\mathrm{e}, i, u}$ for cell-edge users are the same as (19) and (20) for cell-centre users, respectively. Therefore, the same approach to compute the user SIR and other relevant performance metrics for cellcentre users can be readily applied for cell-edge users.

With $N_{\mathrm{a}}<4$, the calculation of SIR $\gamma_{\mathrm{e}, u}$ of user $u$ as a cell-edge user can be simplified as

$$
\gamma_{e, u}=\frac{P_{\mathrm{r}, 1,1, u} \psi_{1, u}}{\sum_{i=2}^{N_{\text {sites }}} P_{\mathrm{r}, i, 1, u} \psi_{i, u}}=\frac{\mathcal{P}_{1,1, u}}{\sum_{i=2}^{N_{\text {sites }}} I_{\mathrm{e}, i, u}},
$$

where $I_{\mathrm{e}, i, u}$ is calculated as

$$
I_{\mathrm{e}, i, u}=P_{\mathrm{r}, i, 1, u} \psi_{i, u} .
$$

$\mu_{I_{\mathrm{e}, i, u}}$ and $\sigma_{I_{\mathrm{e}, i, u}}$ are the mean and standard deviation of the normal distribution associated with the log-normal variable $I_{e, i, u}$, which are calculated as

$$
\mu_{I_{\mathrm{e}, i, u}}=\ln \left(\sum_{j=1}^{N_{\mathrm{a}}} P_{\mathrm{r}, i, j, u}\right), \quad i=2, \ldots, N_{\text {sites }} .
$$

and

$$
\sigma_{I_{\mathrm{e}, i, u}}=\sigma_{\mathrm{w}}
$$

Let us now define $Y_{\text {sum,e, } u}$ as the sum interference from neighbouring cells to signal ratio for cell-edge user $u$, i.e.,

$$
Y_{\mathrm{sum}, \mathrm{e}, u}=\frac{I_{\mathrm{sum}, \mathrm{e}, u}}{\mathcal{S}_{u}}=\frac{1}{\gamma_{\mathrm{e}, u}}
$$

where $I_{\text {sum,e, } u}$ is the sum of the interference from the neighbouring cells to cell-edge user $u$, and can be calculated as $I_{\text {sum }, \mathrm{e}, u}=\sum_{i=2}^{N_{\text {sites }}} I_{\mathrm{e}, i, u}$. Then, as for cell-centre users in the previous subsection, $I_{\mathrm{sum}, \mathrm{e}, u}$ can be approximated by a log-normal RV where $\mu_{I_{\mathrm{sum}, \mathrm{e}, u}}$ and $\sigma_{I_{\mathrm{sum}, \mathrm{e}, u}}$ denote the mean and standard deviation of the normal distribution assocaited with $I_{\text {sum,e }, u}$, respectively, which can be determined using the same approach as for determining (25) and (26), respectively,

With the approximation of $I_{\mathrm{sum}, \mathrm{e}, u}$ as a log-normal RV and because $\mathcal{S}_{u}$ is also a log-normal RV, $Y_{\text {sum,e }, u}$ and $\gamma_{\mathrm{e}, u}$ are also known to be log-normal RVs. Let $\mu_{Y_{\text {sum }, e, u}}$ and $\sigma_{Y_{\text {sum }, e, u}}$ denote the mean and standard deviation of the normal distribution associated with $Y_{\text {sum }, \mathrm{e}, u}$, respectively, which can be determined using the same approach as for determining (29) and (30), respectively.

Let $\mu_{\gamma_{\mathrm{e}, u}}$ and $\sigma_{\gamma_{\mathrm{e}, u}}$ denote the mean and standard deviation of the normal distribution associated with the log-normal RV $\gamma_{\mathrm{e}, u}$. According to the properties of the log-normal RVs, we can write that $\mu_{\gamma_{\mathrm{e}, u}}=-\mu_{Y_{\mathrm{sum}, \mathrm{e}, u}}$ and $\sigma_{\gamma_{\mathrm{e}, u}}=\sigma_{Y_{\mathrm{sum}, \mathrm{e}, u}}$. Then, the mean of $\gamma_{\mathrm{e}, u}$ denoted by $\overline{\gamma_{\mathrm{e}, u}}$ for cell-edge user $u$ can be calculated as

$$
\overline{\gamma_{\mathrm{e}, u}}=\mathrm{e}^{\mu_{\gamma_{\mathrm{e}, u}}+\sigma_{\gamma_{\mathrm{e}, u}}^{2} / 2} .
$$

Based on the previous derivations, the throughput $\mathcal{X}_{\mathrm{e}, u}$ that can be achieved by user $u$ as cell-edge user can be calculated using (11).

a) Outage probability: The outage probability $\mathcal{O}_{\mathrm{e}, u, \gamma_{\text {thr }}}$ for a cell-edge user $u$ with a given SIR threshold $\gamma_{\text {thr }}$ can be calculated as

$$
\begin{aligned}
\mathcal{O}_{\mathrm{e}, u, \gamma_{\mathrm{thr}}} & =\operatorname{Prob}\left(\gamma_{\mathrm{e}, u}<\gamma_{\mathrm{thr}}\right) \\
& =\int_{0}^{\gamma_{\mathrm{thr}}} \frac{1}{y \sigma_{\gamma_{\mathrm{e}, u}} \sqrt{2 \pi}} \mathrm{e}^{-\frac{\left[\ln (y)-\mu_{\gamma_{\mathrm{e}}, u}\right]^{2}}{2 \sigma_{\gamma_{\mathrm{e}}, u}}} d y \\
& =\Phi\left(\frac{\ln \left(\gamma_{\mathrm{thr}}\right)-\mu_{\gamma_{\mathrm{e}, u}}}{\sigma_{\gamma_{\mathrm{e}, u}}}\right),
\end{aligned}
$$

where $\Phi(x)$ is the cumulative distribution function of the standard normal distribution. 


\section{System-level performance analysis}

Due to the symmetry of the sector structure and the full load traffic assumption, it is expected that all sectors have very similar performances. Therefore, the analysis of a representative sector (say sector $\mathcal{A}_{1,1}$ ) is sufficient for system-level performance analysis.

In order to facilitate the analytical evaluation of the systemlevel performance, the whole sector $\mathcal{A}_{1,1}$ service area is divided into segments with equal size of $d_{\text {res }} \times d_{\text {res }} m^{2}$ by equally spaced horizontal and vertical lines. It is noted that the segmentation approach is only applied to the analytical model for theoretic analysis purpose. In the simulations, the users are randomly distributed following a uniform distribution. The segments are given IDs $(k, l), k$ denoting the row number and $l$ denoting the column number associated with the segments. Segment $(0,0)$ corresponds to the top left segment. Let each segment with ID $(k, l)$ be represented by its centre point (denoted by $M_{k, l}$ ), which has a location $L_{M_{k, l}}$. For modelling purpose, the users are randomly located at the centres of the segments with uniform distribution, and are associated to only one sector according to their received signal strength. For simplicity, we have assumed that users are uniformly distributed in the network, but the model can work with any other user distribution.

Let us recall that $\Omega_{\mathrm{c}}$ and $\Omega_{\mathrm{e}}$ denote the sets of cell-centre and cell-edge users in sector $\mathcal{A}_{1,1}$, respectively, where $N_{\mathrm{c}}$ and $N_{\mathrm{e}}$ denote their cardinality, respectively. As the locations of users in sector $\mathcal{A}_{1,1}$ are known, the sets $\Omega_{\mathrm{c}}$ and $\Omega_{\mathrm{e}}$ can be determined according to the user classification methods described in Section III. Specifically for the traditional IFR-1 scheme $\Omega_{\mathrm{e}}=\phi$ and $N_{\mathrm{e}}=0$, while for the traditional IFR-3 scheme $\Omega_{\mathrm{c}}=\phi$ and $N_{\mathrm{c}}=0$. Then, we can approximate the system-level performance as follows.

Let $\gamma_{\text {net }}$ denote the mean SIR of the entire network,

$$
\gamma_{\text {net }}=\frac{\sum_{u \in \Omega_{\mathrm{c}}} \gamma_{\mathrm{c}, u}+\sum_{u \in \Omega_{\mathrm{e}}} \gamma_{\mathrm{e}, u}}{N_{\mathrm{c}}+N_{\mathrm{e}}},
$$

and

$$
\gamma_{\text {net }, \mathrm{db}}=\frac{\sum_{u \in \Omega_{\mathrm{c}}} \gamma_{\mathrm{c}, u, \mathrm{db}}+\sum_{u \in \Omega_{\mathrm{e}}} \gamma_{\mathrm{e}, u, \mathrm{db}}}{N_{\mathrm{c}}+N_{\mathrm{e}}},
$$

where $\gamma_{\mathrm{c}, u}$ and $\gamma_{\mathrm{e}, u}$ are computed by (19) and (36), respectively.

Similarly, we can calculate the site throughput $\mathcal{X}_{\text {site }}$ by (13) and the outage probability $\mathcal{O}_{\text {net }, \gamma_{\text {thr }}}$ of the entire network with respect to a SIR outage threshold $\gamma_{\text {thr }}$ by

$$
\mathcal{O}_{\text {net }, \gamma_{\mathrm{thr}}}=\frac{\sum_{u \in \Omega_{\mathrm{c}}} \mathcal{O}_{\mathrm{c}, u, \gamma_{\mathrm{thr}}}+\sum_{u \in \Omega_{\mathrm{e}}} \mathcal{O}_{\mathrm{e}, u, \gamma_{\mathrm{thr}}}}{N_{\mathrm{c}}+N_{\mathrm{e}}} .
$$

\section{NumericAl RESUlts}

\section{A. System configurations}

In total, twelve system configurations presented in Table II with 3, 6 and 12 sectors per BS are evaluated and compared, using the proposed analytical model as well as systemlevel simulations. Analytical results are obtained using Matlab numerical tools, where simulation results are averaged over 2000 simulations runs. The corresponding bandwidth partition coefficient $\beta$ was set to 0.5 , unless stated otherwise, meaning that cell-centre users benefit from 50\% of the bandwidth. Table III presents the most relevant system parameters.

In the simulations, the users are uniformly distributed within the coverage area of sector $\mathcal{A}_{1,1}$. The user density used in the simulations is 0.0025 active user per square meter.

For the numerical evaluation, we use a spectral efficiency function $\mathcal{F}(x)$ which approximates an abstracted LTE link level model from the Vienna LTE link level Simulator [34], with $2 \times 2$ antenna mode, open loop spatial multiplexing (OLSM) and adaptive modulation and coding (AMC). The original LTE link-level model (presented in Fig. 9 of [34]) mapping channel SNR $(\mathrm{dB})$ to spectral efficiency $(\mathrm{bps} / \mathrm{Hz})$ is approximated by the following spectral efficiency function:

$$
\mathcal{F}(x)= \begin{cases}7, & x>40 \mathrm{~dB} \\ \sum_{i=0}^{6} c_{i} x^{i}, & -10 \leq x \leq 15 \mathrm{~dB} \\ 0 & x<-10 \mathrm{~dB},\end{cases}
$$

where the function input $x$ corresponds to SIR value in $\mathrm{dB}$, and the polynomial coefficients $c_{i}$ are determined by a Matlab curve fitting tool as $0.5935,0.09151,0.001567,0.0001185$, $1.8 \mathrm{e}-05,1.0 \mathrm{e}-06,1.3 \mathrm{e}-08$, for $i=0,1, \ldots, 6$, respectively. The analytical model can be applied to work with other abstracted link level models.

\section{B. Network performance}

Figures 3 and Figures 4 show the network performance in terms of mean user SIR, mean site throughput and mean outage probability, against the various presented system configurations for $R_{\mathrm{ISD}}=500 \mathrm{~m}$ and $R_{\mathrm{ISD}}=1000 \mathrm{~m}$, respectively. In the figure, the 5\%-tile site throughput performance is shown. The $95 \%$ confidence intervals for mean user SIR, mean site throughput and mean outage probability are also presented. From these results, it can be observed that analytical results closely match system-level simulation results. In more detail, the average difference between analytical and system-level simulation results is less than 5\%. This fact shows the accuracy of our proposed analytical model, one of the main contribution of this paper. This accuracy enables the use of this analytical tool as an effective method to predict network performance in a fast and reliable manner. For example, optimization tools that attempt to find a proper antenna orientation and downtilt in HOS deployments can use the proposed analytical model to quickly search over different candidate configurations and find the best performing one.

Table IV and Table V provide more detailed information about the mean site throughput and mean outage probability for the different system configurations, indicating the relative performance gains with respect to S3/IFRI. In Tables IV and $\mathrm{V}$, the rows with labels 'Mod' and 'Sim' correspond to the results with path loss and shadowing fading only (modelled and simulated, respectively); while the rows with labels 'SimSmall correspond to the results with path loss, shadowing fading and fast fading (simulated). The fast fading follows 
TABLE II

SYSTEM CONFIGURATION NAMES AND DETAILS

\begin{tabular}{|c|c|c|c|c|c|c|c|c|c|c|c|c|}
\hline Config. & S3/IFRI & S6/IFRI & S12/IFR1 & S3/SE & $S 6 / S E$ & S12/SE & S3/RSS & S6/RSS & S12/RSS & S3/DIST & S6/DIST & S12/DIST \\
\hline \#. sectors & 3 & 6 & 12 & 3 & 6 & 12 & 3 & 6 & 12 & 3 & 6 & 12 \\
\hline Classifica. & & N/A & & & UC-Spe & & & UC-RSS & & & UC-Dist & \\
\hline Reuse & & IFR-1 & & & & & & $\mathrm{FF}$ & & & & \\
\hline
\end{tabular}

TABLE III

SYSTEM SETTINGS

\begin{tabular}{l|l}
\hline Parameters & Value \\
\hline Carrier frequency & $2000 \mathrm{MHz}$ \\
Bandwidth & $5 \mathrm{MHz}$ \\
Number of sites & $N_{\text {sites }}=19$ \\
Inter-site distance & $R_{\mathrm{ISD}}=500 \mathrm{~m}, 1000 \mathrm{~m}$ \\
Number of sectors & $N_{\mathrm{a}}=3,6,12$ \\
Transmit power & $21.6 \mathrm{~W}$ \\
Shadowing standard deviation & $6 \mathrm{~dB}$ \\
Shadow correlation & $\rho=0.5$ \\
Antenna height & $25 \mathrm{~m}$ \\
Max antenna gain $(\mathrm{dBi})$ & $15.5 / 19.8 / 22(3 / 6 / 12 \mathrm{sec})$. \\
Antenna front to back ratio & $25 \mathrm{~dB}$ \\
Horizontal HPBW $\vartheta_{3 \mathrm{~dB}}$ & $65^{\circ} / 33^{\circ} / 17^{\circ}(3 / 6 / 12 \mathrm{sec})$. \\
Antenna mode & $2 \times 2 \mathrm{antennas}$ \\
Vertical HPBW $\theta_{3 \mathrm{~dB}}$ & $11.5^{\circ} / 8.5^{\circ} / 8.5^{\circ}(3 / 6 / 12 \mathrm{sec})$. \\
Segment resolution $d_{\mathrm{res}}$ & $10 \mathrm{~m}$ \\
Antenna downtilt $\theta_{\text {down }}$ & $10.38^{\circ} / 8.28^{\circ} / 8.28^{\circ}(3 / 6 / 12 \mathrm{sec})$. \\
User density (simulation) & $0.0025 \mathrm{per} \mathrm{m}^{2}$ \\
Outage threshold $\gamma_{\text {thr }}$ & $-5 \mathrm{~dB}$ \\
Bandwidth partition coefficient $\beta$ & {$[0,0.1, \ldots, 1]$} \\
\hline
\end{tabular}

a Rayleigh distribution with scale parameter set to $2 / \sqrt{\pi}$ to have a mean value of 1 for the Rayleigh distribution. It can be observed that there is a small difference between the throughput results obtained without and with fast fading under the investigated system settings, mainly due to the averaging over a large number of snapshots. There is a small increase in outage probability when fast fading is considered. As the fast fading impact is negligible in this scenario, fast fading is not considered in the simulations in the remaining of the paper.

Moreover, it is noted that mean deviation of mean site throughputs with $U C$-Spe-Acc and UC-Spe-App methods was less than $1.4 \%$ indicating that our approximation is accurate. As a consequence, results with UC-Spe-Acc method are not shown in this section for the sake of space.

a) Impact of the number of sectors per BS on system performance: If we compare the configurations with 3,6 and 12 sectors per BS, it can be observed from Fig. 3 that:

- Average network SIR decreases with increasing number of sectors per BS. This is due to the larger inter-cell interference introduced by more sectors per BS.

- Average site throughput increases with increasing number of sectors per BS. This is due to the lager spatial reuse introduced by more sectors per BS, which outweighs the SIR decrease.

However, it is important to note that doubling the number of sectors per BS does not double the site throughput. Indeed, when we move from $S 3 / I F R 1$ to $S 6 / I F R 1$, the average site throughput increases by $71 \%$, while when we move from S6/IFRI to S12/IFR1, the average site throughput increases by $50 \%$ (see Table IV for detail analysis of average site throughput gains). This indicates that there is a diminishing

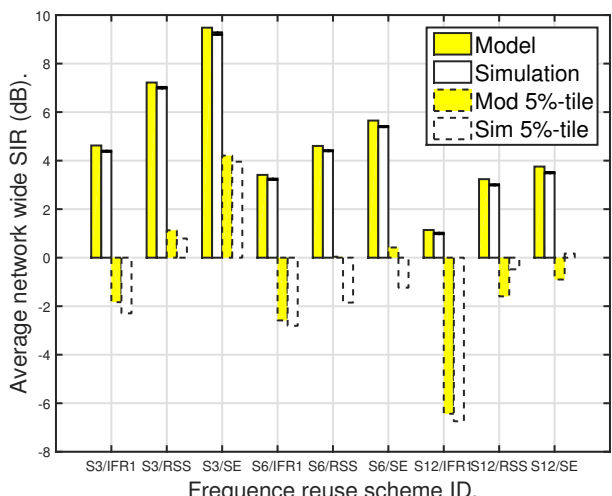

(a) Mean SIR (dB).

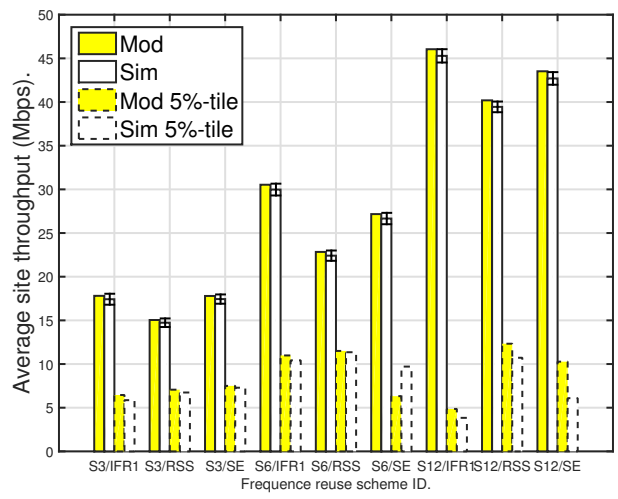

(b) Mean site throughput (Mbps)

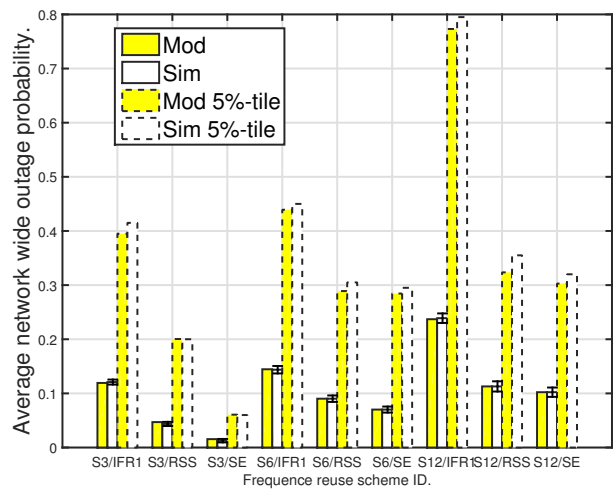

(c) Mean outage probability.

Fig. 3. Network wide performances with $R_{\mathrm{ISD}}=500 \mathrm{~m}$, bandwidth coefficient $\beta=0.5$.

performance gain with the increasing number of sectors per BS due to interference, and adding more sectors per BS may not always increase the average network throughput in a cost effective manner. Similar trends are observed for Fig. 4 with $R_{\mathrm{ISD}}=1000 \mathrm{~m}$, and the other user classification methods. This highlights the importance of frequency reuse schemes, 
TABLE IV

MEAN SITE THROUGHPUT AND GAIN OVER S3/IFRI

\begin{tabular}{lllllllllll}
\hline Setting & & \multicolumn{9}{c}{ Throughput (Mbp) and gains over S3/IFRI (\%) } \\
\hline$R_{\mathrm{ISD}} / \beta$ & & S3/IFRI & S3/RSS & S3/SE & S6/IFRI & S6/RSS & S6/SE & S12/IFRI & S12/RSS & S12/SE \\
\hline $500 / 0.3$ & Mod & $17.8 / 0.0$ & $15.0 /-15.5$ & $17.8 /-0.1$ & $30.5 / 71.4$ & $22.8 / 28.2$ & $27.2 / 52.6$ & $46.0 / 158.6$ & $40.2 / 125.7$ & $43.5 / 144.4$ \\
& Sim & $17.4 / 0.0$ & $14.7 /-15.4$ & $17.4 / 0.1$ & $30.0 / 72.1$ & $22.4 / 28.6$ & $26.7 / 53.0$ & $45.3 / 160.0$ & $39.4 / 126.4$ & $42.7 / 145.1$ \\
& Sim-Small & $17.4 / 0.0$ & $14.8-15.0$ & $17.5 / 0.7$ & $29.7 / 70.7$ & $22.3 / 28.1$ & $26.6 / 52.6$ & $44.6 / 156.2$ & $39.1 / 124.4$ & $42.3 / 143.0$ \\
\hline $1000 / 0.3$ & Mod & $15.2 / 0.0$ & $12.7 /-16.3$ & $15.1 /-0.8$ & $26.3 / 73.2$ & $20.2 / 33.0$ & $23.7 / 56.2$ & $40.2 / 164.4$ & $37.2 / 144.8$ & $39.0 / 156.8$ \\
& Sim & $14.9 / 0.0$ & $12.5 /-16.1$ & $14.8 /-0.3$ & $25.8 / 73.6$ & $19.8 / 33.2$ & $23.4 / 56.9$ & $39.5 / 165.4$ & $36.5 / 145.0$ & $38.3 / 157.4$ \\
& Sim-Small & $14.9 / 0.0$ & $12.6 /-15.7$ & $14.9 / 0.0$ & $25.7 / 72.3$ & $19.8 / 32.8$ & $23.3 / 56.2$ & $38.9 / 161.0$ & $36.1 / 142.3$ & $37.9 / 154.6$ \\
\hline
\end{tabular}

TABLE V

MEAN OUTAGE PROBABILITY AND GAIN OVER S3/IFRI

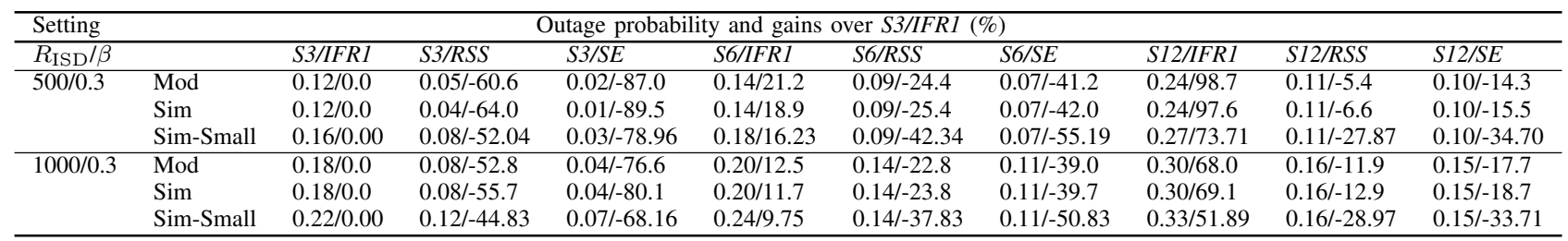

which can be an important solution to the above problem by achieving linear throughput gains while maintaining relatively low outage probability.

b) Impact of user classification methods on system performance: If we compare the configurations with different user classification methods, it can also be observed from Fig. 3 that:

- Due to the lack of inter-cell interference coordination, IFRI provides the worst mean and 5\% tile SIR performance, which translates to the largest mean outage probability. Indeed, IFRI results in a high mean outage probability of $12 \%$ for the 3 sector case and $24 \%$ for the 12 sector case. As a note, it is interesting to observe that IFRI outperforms RSS in terms of mean average throughput. This is because the mean throughput of IFRI is driven by its cell-centre users, which benefit from the larger available bandwidth in IFRI than in RSS. However, the better inter-cell interference coordination of RSS results in a better 5\%-tile throughput.

- Due to inter-cell interference coordination and the enhanced user classification method, $S E$ provides the best mean and 5\%tile SIR performance, which is translated into the lowest mean outage probability. $S E$ decreases the mean outage probability with respect to RSS by around $73 \%$ for the 3 sector case and $22 \%$ for the 6 sector case. This shows the better fairness of the proposed user classification method. In $S E$, all users receive the same amount of bandwidth regardless of their positions, and as a by-product more users are classified as cell-edge users and thus benefit from inter-cell interference coordination. In terms of mean average throughput, $S E$ significantly outperforms RSS with gains of $19 \%$ for both 3 sector and 6 sector cases, at the expense of some slight degradation on 5\%-tile throughput for the 6 and 12 sector cases. As mentioned before, since more users are classified as cell-edge users, the outage probability decreases, but also the 5\%-tile throughput. Note that this trade-offs can be controlled by using the bandwidth partition coefficient $\beta$, as it is shown in the next section.
The different system configurations with $R_{\mathrm{ISD}}=1000 \mathrm{~m}$ follow the same trends, as shown in Fig. 4.

\section{Impact of bandwidth partition on FFR performances}

In the following, a study is performed to assess how the different system configurations may perform under different bandwidth partitions to cell-centre and cell-edge users. In order to do this, the bandwidth partition coefficient $\beta$ is varied between 0 and 1 with a step size of 0.1 , and the corresponding site throughput and resource allocation fairness are calculated.

The widely used Jain's fairness index is used to measure the resource allocation fairness [35]. Assume that there are $N_{\mathrm{c}}$ cell-centre users and $N_{\mathrm{e}}$ cell-edge users after running the user classification method, which have been allocated a bandwidth $\beta \mathcal{W}_{\text {all }}$ and $(1-\beta) \mathcal{W}_{\text {all }}$, respectively, and that the available bandwidth in each band is equally shared among the existing users (i.e., round robin). Then, the resource allocation fairness can be calculated using Jain's fairness index [35]. $\zeta(\beta)$ can be calculated as:

$$
\begin{aligned}
\zeta(\beta) & =\frac{\left[\frac{N_{\mathrm{c}} \beta \mathcal{W}_{\text {all }}}{N_{\mathrm{c}}}+\frac{N_{\mathrm{e}}(1-\beta) \mathcal{W}_{\text {all }}}{N_{\mathrm{e}}}\right]^{2}}{\left(N_{\mathrm{c}}+N_{\mathrm{e}}\right)\left\{N_{\mathrm{c}}\left(\frac{\beta \mathcal{W}_{\text {all }}}{N_{\mathrm{c}}}\right)^{2}+N_{\mathrm{e}}\left[\frac{(1-\beta) \mathcal{W}_{\text {all }}}{N_{\mathrm{e}}}\right]^{2}\right\}} \\
& =\frac{1}{\left(N_{\mathrm{c}}+N_{\mathrm{e}}\right)\left[\frac{\beta^{2}}{N_{\mathrm{c}}}+\frac{(1-\beta)^{2}}{N_{\mathrm{e}}}\right]}
\end{aligned}
$$

For this setup, Fig. 5(a) shows the site throughput resulting from system configuration $S 6 / D I S T$ with $\alpha=0.7, S 6 / R S S, S 6 / S E$ and $S 6 / S E-A c c$, against the bandwidth partition coefficient $\beta$ for $R_{\mathrm{ISD}}=500 \mathrm{~m}$. Figure $5(\mathrm{~b})$ presents the corresponding outage probability, while Fig. 5(c) presents the corresponding resource allocation Jain's fairness index. Both simulation and analytical results are presented for site throughput and outage probability, while only simulation results are presented for the fairness index for readability. 


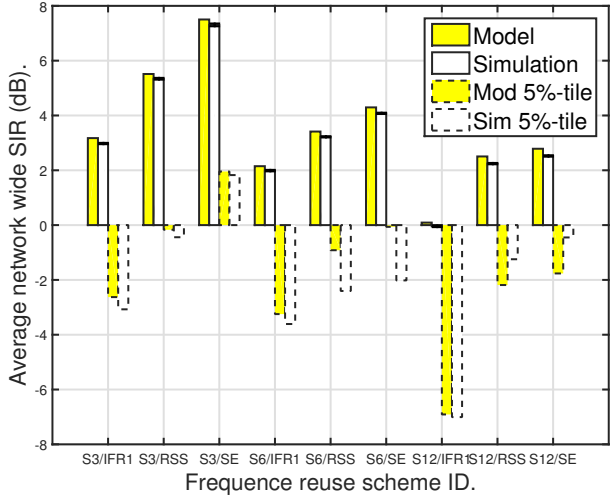

(a) Mean SIR (dB)

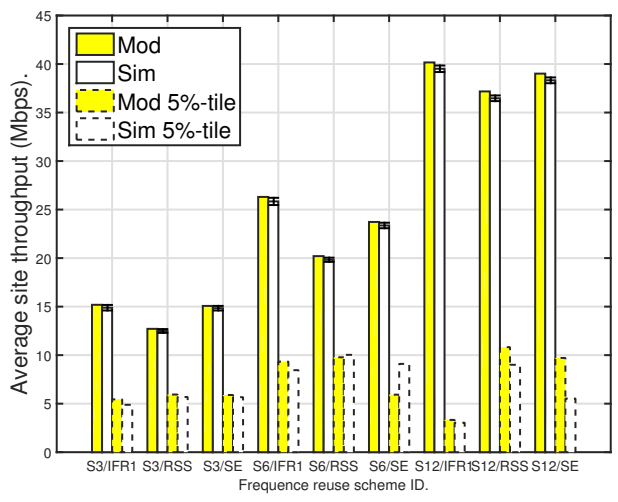

(b) Mean site throughput (Mbps).

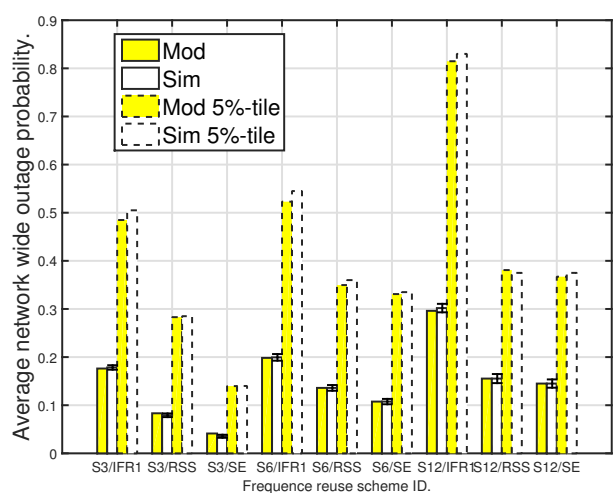

(c) Mean outage probability.

Fig. 4. Network wide performances with $R_{\mathrm{ISD}}=1000 \mathrm{~m}$, bandwidth coefficient $\beta=0.5$.

First of all, it is important to note again that the analytical results closely match system-level simulation results, which demonstrates the accuracy of our analytical model.

In terms of the study, it is noted that $S 6 / S E$ achieves the highest possible fairness index value for all bandwidth partition coefficients $\beta$, meaning that all users receive the same amount of bandwidth regardless of their positions and the bandwidth partition coefficient. This mitigates the impact of the user distribution and dynamics on the system performance. This was a design objective, which translates to a fairly comparable site throughput for all values of $\beta$. In more detail, $S 6 / S E$ maintains a relatively high network throughput, which monotonically and slowly increases with $\beta$. Looking at the extreme cases, if $\beta=0$, all the bandwidth is cell-edge bandwidth and all users are classified as cell-edge users. Thus the system suffers from resource muting. If $\beta=1$, all the bandwidth is cell-centre bandwidth and all users are classified as cell-centre users. Thus the system suffers from inter-cell interference. According to our results, $\beta=0.5$ is a good operation point for this scenario, with close to the highest throughput and lowest outage probability.

In contrast, for $S 6 / D I S T$ and $S 6 / R S S$, since user classification is fixed and does not depend on $\beta$ (but on distances and received signal strength, respectively), the fairness index significantly varies with $\beta$. As a result, all users do not always receive the same amount of bandwidth in the scenario, and thus the user distribution and dynamics as well as the bandwidth partition have a significant impact on system performance. For example, with regard to user distribution, if at a given point in time there are no cell-edge users according to the S6/DIST and S6/RSS user classification methods, the cell-edge bandwidth would be unused. This is inefficient and does not occur with $S E$, which has the capability to classify a user as cell-centre or cell-edge user on the fly according to traffic conditions. With regard to bandwidth partitioning, it is also important to note that for $S 6 / D I S T$ and $S 6 / R S S$, as a result of the fix user classification, the larger the $\beta$, the more resources are allocated to cell-centre users, and thus the larger is the resulting site throughput at the expense of a decreased celledge performance. As a result, the site throughputs of S6/DIST and S6/RSS monotonically increase with $\beta$, at the expense of fairness. It is important to note that with $\beta=0.6$ the fairness indices of S6/RSS and S6/DIST are close to 1 , and the site throughput of $S 6 / R S S$ and S6/DIST are similar to that of S6/SE. However, the outage probabilities of S6/RSS and S6/DIST are much higher.

\section{CONCLUSION}

In this paper, HOS deployments have been considered as a cost-effective approach to improve network capacity, and their performances have been analysed in detail. Towards this objective, a novel statistical model has been presented to theoretically characterize the HOS deployment performance in conjunction with a strict FFR scheme in terms of user signal quality, site throughput and outage probability. Moreover, we have considered the impact of different user classification methods in the FFR scheme, and proposed a new spectrum efficiency-based user classification method that improves resource utilization and allocation fairness. It is important to note that the proposed statistical model can be easily extended to work with different BS placements and user distribution, as well as scheduling techniques. Our studies show that the average difference between analytical and system-level simulation results are less than 5\% in terms of mean user SIR, mean site throughput, and mean outage probability, indicating a good accuracy of the proposed analytical model. In terms of mean site throughput, HSO provide a diminishing gain with the number of antennas due to inter-cell interference. Performance results also show that the proposed user classification method, $S E$, outperforms the RSS method with gain of $19 \%$ for both 3 -sectors and 6-sectors cases. Moreover, it decreases outage 


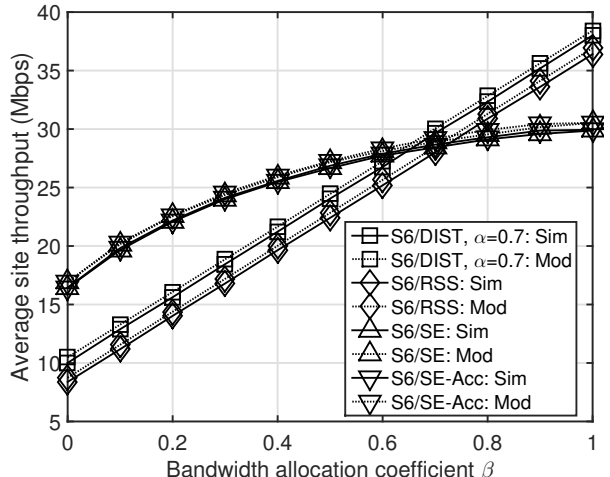

(a) Mean site throughput (Mbps).

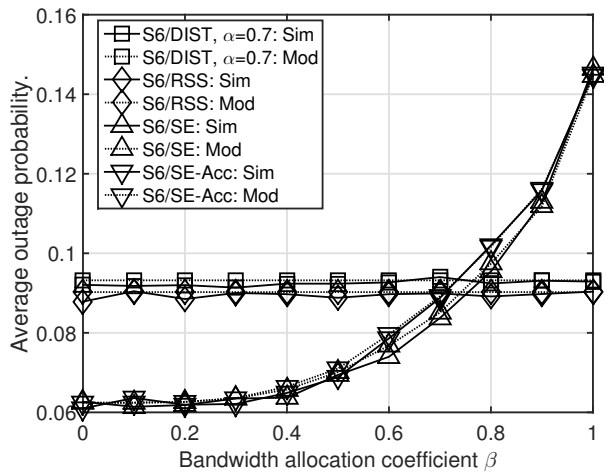

(b) Mean outage probability.

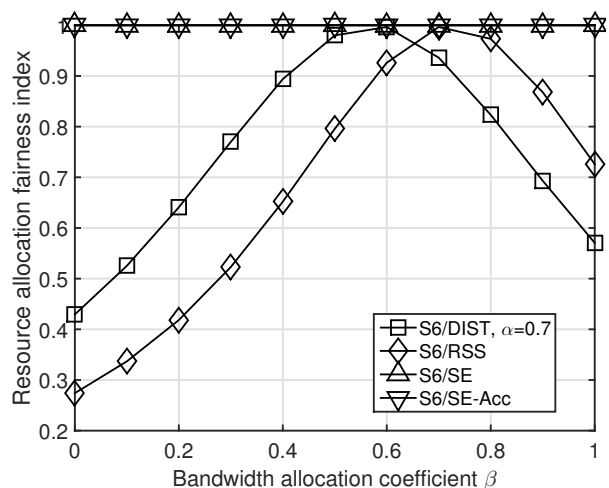

(c) Fairness index on resource allocation.

Fig. 5. Network performance against bandwidth partition coefficient with $R_{\mathrm{ISD}}=500 \mathrm{~m}$.

probability with respect to the $R S S$ method by around $73 \%$ for the 3 sector case and $22 \%$ for the 6 sector case. In the future, we are interested in investigating other FFR schemes and interference coordination schemes to HOS deployments. In addition we are interested in the investigation on the combination of azimuth and vertical sectorization to find out efficient settings to improve network performance.

\section{ACKNOWLEDGEMENT}

The authors would like to thank the anonymous reviewers for their constructive comments, which helped improve the quality of this paper.

\section{REFERENCES}

[1] H. Holma and A. Toskala, "LTE for UMTS: evolution to LTEAdvanced," John Wiley \& Sons Ltd, 20011.
[2] C. Wang et al, "Cellular architecture and key technologies for $5 \mathrm{G}$ wireless communication networks," IEEE Commun. Mag., Vol. 52, No. 2, pp.122-130, Feb. 2014.

[3] N. Lee et al, "Spectral efficiency of dynamic coordinated beamforming: a stochastic geometry approach," IEEE Trans. Wireless Commun., Vol. 14, No. 1, pp. 230-241, Jan. 2015.

[4] A.S. Hamza et al, "A survey on inter-cell interference coordination techniques in OFDMA-based cellular networks," IEEE Commun. Surveys \& Tutorials, Vol. 15, No. 4, pp. 1642-1670, 2013.

[5] X. Zhang, M. Haenggi, "A Stochastic Geometry analysis of inter-cell interference coordination and intra-cell diversity," IEEE Trans. Wireless Commun., Vol. 13, No. 12, pp. 6655-6669, Dec. 2014.

[6] 3GPP TR 36.814 V9.0.0, "Further advancements for E-UTRA physical layer aspects," Technical Report, March 2010.

[7] J. Ylitalo and E. Tiirola "Performance evaluation of different antenna array approaches for 3G CDMA uplink," in Proc. IEEE VTC'00, 2000.

[8] B. Hagerman et al, "WCDMA 6-sector deployment- case study of a real installed UMTS-FDD network," in Proc. of IEEE VTC'06, 2006.

[9] A. Osseiran and A. Logothetis, "Smart antennas in a WCDMA radio network system: modeling and evaluations," IEEE Trans. Antennas and Propagation, Vol. 54, No. 11, pp. 3302-3316, Nov. 2006.

[10] A. Osseiran, P. Skillermark and M. Olsson, "Multi-antenna SDMA in OFDM radio networks systems: modeling and evaluations," in Proc. of IEEE PIMRC'07, 2007.

[11] H. Huang et al, "Increasing downlink cellular throughput with limited network MIMO coordination," IEEE Trans. Wireless Commun., Vol. 8 , No. 6, pp. 2983-2989, June 2009.

[12] H. Huang et al, "Increasing throughput in cellular networks with higherorder sectorization," in Proc. Asilomar'10, 2010

[13] I. Riedel and G. Fettweis, "Increasing throughput and fairness in the downlink of cellular systems with N-fold sectorization," in Proc. of IEEE GlobeCom'11, 2011.

[14] R. Joyce and L. Zhang, "Higher order horizontal sectorisation gains for a real 3GPP/HSPA+ network," in Proc. of European Wireless, 2013.

[15] W. Guo and T. O'Farrell, "Dynamic cell expansion with self-organizing cooperation ", IEEE Journal on Selected Areas in Commun., Vol.31, No. 5, pp. 851-860, April 2013.

[16] M. Sheikh,, and J. Lempiinen, "A flower tessellation for simulation purpose of cellular network with 12-sector sites," IEEE Wireless Commun. Lett., Vol. 2, No. 3, pp. 279-282, June 2013.

[17] H. Claussen, "Efficient modelling of channel maps with correlated shadow fading in mobile radio systems," in Proc. of IEEE PIMRC'05, September 2005.

[18] D. Lopez-Perez, H. Claussen, L. Ho, "Improved frequency reuse schemes with horizontal sector offset for LTE," in Proc. of IEEE PIMRC'13, 2013.

[19] S. Ali, V. Leung, "Dynamic frequency allocation in fractional frequency reused OFDMA networks," IEEE Trans. Wireless Commun., Vol. 8, No. 8, pp. 4286-4295, 2009.

[20] T. Novlan, R. Ganti, A. Ghosh, J. Andrews "Analytical evaluation of fractional frequency reuse for OFDMA cellular networks," IEEE Trans. Wireless Commun., Vol. 10, No. 12, pp. 4294-4305, 2011

[21] L. Chen et al, "System-level simulation methodology and platform for mobile cellular systems," IEEE Commun. Mag., Vol. 49, No. 7, 2011.

[22] F. Baccelli, A. Giovanidis, "A stochastic geometry framework for analyzing pairwise-cooperative cellular networks," IEEE Trans. Wireless Commun., Vol. 14, No. 2, pp. 794-808, Feb. 2015.

[23] T. Bai, R.W. Heath, "Coverage and rate analysis for millimeter-wave cellular networks," IEEE Trans. Wireless Commun., Vol. 14, No. 2, pp. 1100-1114, Feb. 2015.

[24] L. Fenton, "The sum of log-normal probability distributions in scatter transmission systems," IEEE Trans. Commun., Vol. 8, No. 1, pp. 57-67, March 1960.

[25] N. B. Mehta, A. F. Molisch, J. Wu and J. Zhang, "Approximating the sum of sorrelated Lognormal or, Lognormal-Rice random variables," In Proc. IEEE ICC'06, pp. 1605-1610, 2006,

[26] A. Safak, "Statistical analysis of the power sum of multiple correlated log-normal components," IEEE Trans. Veh. Technol., Vol. 42, No. 1, pp. 58-61, Feb. 1993

[27] S. Schwartz and Y. Yeh, "On the distribution function and moments of power sums with lognormal components," Bell System Technology Journal, Vol. 61, No. 7, pp. 1441-1462, September 1982.

[28] A. Abu-Dayya and N. Beaulieu, "Outage probabilities in the presence of correlated lognormal interferers, IEEE Trans. Veh. Technol., Vol. 43, No. 1, pp. 164-173, Feb. 1994. 
[29] N. Mehta, J. Wu, A. Molisch, J. Zhang "Approximating a sum of random variables with a Lognormal," IEEE Trans. Wireless Commun., Vol. 6, No. 7, pp. 2690-2699, July 2007.

[30] T. Bonald and A. Proutiere, "Wireless downlink data channels: user performance and cell dimensioning," In Proc. Mobicom'03, 2003.

[31] J. Andrews, F. Baccelli, and R. Ganti, "A tractable approach to coverage and rate in cellular networks," IEEE Trans. Commun., Vol. 59, No. 11, pp. 3122-3134, Nov. 2011.

[32] Y. Cho, E. Oh, D. Hong, "A new modeling scenario and analysis of handoff algorithms in multisector systems with cochannel interference," IEEE Trans. Veh. Technol., Vol. 58, No. 3, pp. 1407-1414, March 2009.

[33] A. Ligeti, "Outage probability in the presence of correlated lognormal useful and interfering components," IEEE Commun. Lett., Vol. 4, No. 1, pp. 15-17, Jan. 2000.

[34] Mehlfuhrer et al, "The Vienna LTE simulators - enabling reproducibility in wireless communications research," EURASIP Journal on Advances in Signal Processing, pp.1-14, 2011:29, 2011.

[35] D. Chiu, R. Jain, "Analysis of the increase and decrease algorithms for congestion avoidance in computer networks," Computer Networks and ISDN systems, Vol. 17, No. 10, pp. 1-14, 1989.

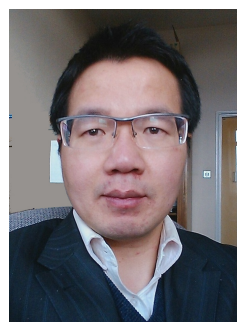

Dr Jianhua He is a Lecturer at Aston University, UK. He received his BSc and MSc degrees from Huazhong University of Science and Technology (HUST), China, and $\mathrm{PhD}$ degree from Nanyang Technological University, Singapore, in 1995, 1998 and 2002, respectively. Dr He was an Associate Professor of Department of Electronics and Information Engineering since 2001. He was with University of Bristol from 2004 to 2006 and with University of Essex in 2007. His main research interests include $5 \mathrm{G}$ and beyond technologies, machine to machine communications, Internet of things system and technologies (e.g. for small cities and intelligent transport) and big data analytics. He has authored or co-authored over 150 technical papers in major international journals and conferences. Dr He is a Senior Member of IEEE. He is editor/guest editor of a number of journals and TPC chair of several international conferences. He was TPC member of many international conferences including IEEE Globecom and IEEE ICC.

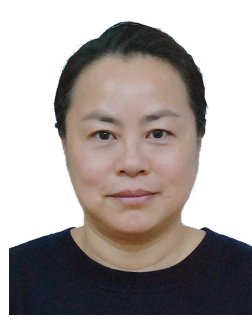

Dr Wenqing Cheng received the B.S. degree in Telecommunication Engineering in 1985 and Ph.D. in Electronics and Information Engineering in 2005, both from Huazhong University of Science and Technology, Wuhan, China. She is currently a professor with the School of Electronic Information and Communications, Huazhong University of Science and Technology. Her research interests include mobile communications and wireless sensor networks, information system, e-Learning applications and etc.

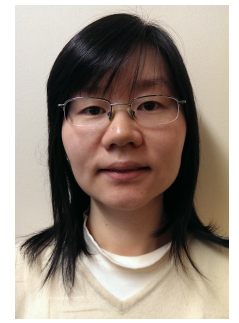

Dr Zuoyin Tang is currently a Lecturer in the School of Engineering and Applied Science, Aston University, UK. She obtained her PhD degree from University of Bath, UK, in 2008. She has authored and co-authored over 40 technical papers in major international journals and conferences. Dr Tang's main research interests include resource management for cellular networks, Internet of things and wireless sensor networks.

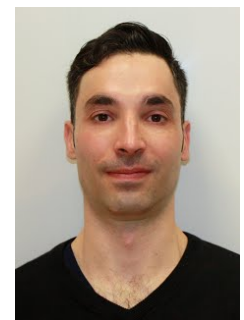

Dr David Lopez-Perez is a Member of Technical Staff at Nokia Bell Laboratories, and his main research interests are in HetNets, small cells, massive MIMO, unlicensed technologies interference and mobility management as well as network optimization and simulation. Prior to this, David earned his $\mathrm{PhD}$ in Wireless Networking from the University of Bedfordshire, UK in Apr. 11, and obtained his BSc and MSc degrees in Telecommunication from the Miguel Hernandez University, Spain in Sept. 03 \& Sept. 06, respectively. David was Research Associate at King's College London, UK from Aug. 10 to Dec. 11, carrying postdoctoral studies, and was with VODAFONE, Spain from Feb. 05 to Feb. 06, working in the area of network planning and optimization. David was also invited researcher at DOCOMO USA labs, CA in 2011, and CITI INSA, France in 2009. For his publications and patent contributions, David is a recipient of both the Bell Labs Alcatel-Lucent Award of Excellence and Certificate of Outstanding Achievement. He was also finalist for the Scientist of the Year prize in The Irish Laboratory Awards (2013, 2015). David was also awarded as PhD Marie-Curie Fellow in 2007 and Exemplary Reviewer for IEEE Communications Letters in 2011. David is author of the book "Heterogeneous Cellular Networks: Theory, Simulation and Deployment" Cambridge University Press, 2012, and has published more than 80 book chapters, journal and conference papers, all in recognized venues, and filed more than 30 patents applications. David is or has been guest editor of a number of journals, e.g., IEEE JSAC, IEEE Comm. Mag., TPC member of top tier conferences, e.g., IEEE Globecom and IEEE ICC, and co-chair of a number of workshops.

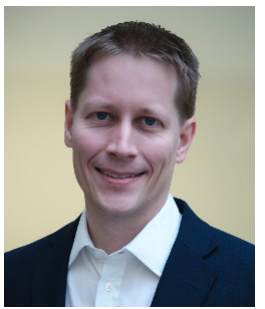

Dr Holger Claussen is leader of Small Cells Research Department at Bell Labs, Nokia with a team in Ireland and the US. In this role, he and his team are innovating in all areas related to future evolution, deployment, and operation of small cell networks to enable exponential growth in mobile data traffic. His research in this domain has been commercialized in Nokias (formerly Alcatel-Lucents) Small Cell product portfolio and continues to have significant impact. He received the 2014 World Technology Award in the individual category Communications Technologies for innovative work of the greatest likely long-term significance. Prior to this, Holger was head of the Autonomous Networks and Systems Research Department at Bell Labs Ireland, where he directed research in the area of self-managing networks to enable the first large scale femtocell deployments from 2009 onwards. Holger joined Bell Labs in 2004, where he began his research in the areas of network optimization, cellular architectures, and improving energy efficiency of networks. Holger received his Ph.D. degree in signal processing for digital communications from the University of Edinburgh, United Kingdom in 2004. He is author of more than 90 publications and 110 filed patent applications. He is Fellow of the World Technology Network, senior member of the IEEE, and member of the IET 